PROCEEDINGS OF THE

AMERICAN MATHEMATICAL SOCIETY

Volume 128, Number 12, Pages 3723-3729

S 0002-9939(00)05732-4

Article electronically published on June 7, 2000

\title{
A NOTE ON PONTRJAGIN FORMS
}

\author{
MAHUYA DATTA
}

(Communicated by Christopher Croke)

\begin{abstract}
Let $P$ be a principal $O(2 m)$ bundle over a manifold $M$ of dimension $4 m$, and let $p$ be its $m$-dimensional Pontrjagin class. In this paper, we aim at answering the following question: Which representatives of the class $p$ can be realised as the Pontrjagin form of some connection on $P$ ?
\end{abstract}

\section{INTRODUCTION}

Let $M$ be a smooth $4 m$-dimensional manifold and let $P \longrightarrow M$ be a smooth principal bundle with structure group $G=O(n)$, where $n \geq 2 m$. Let $p(P) \in$ $H^{4 m}(M)$ denote the $m$-dimesnional Pontrjagin class of the bundle $P$. It would be interesting to know which forms representing the Pontrjagin class $p(P)$ can be realized by some connection on $P$.

Recall that if $\omega$ is a connection form on the principal bundle $P$, then its $m$ dimensional Pontrjagin form is $\operatorname{tr}\left((D \omega)^{2 m}\right)$, where $D \omega$ denotes the covariant derivative of $\omega$. Therefore, if a $4 m$-form $\sigma$ represents the Pontrjagin class $p(P)$ for some bundle $P$, then necessarily there would exist a $\mathfrak{g}$-valued 2 -form $\Omega$ on $M$ of $A d G$-type satisfying $\operatorname{tr}\left(\Omega^{2 m}\right)=\sigma$.

In this paper we prove that when $M$ is an open orientable manifold then this condition is, in fact, sufficient. We state our main result in the following

Theorem 1.1. Let $M$ be a $4 m$-dimensional smooth orientable open manifold. Let $\sigma$ be a volume form on $M$. If there exists a $\mathfrak{g}$-valued 2 -form $\Omega$ on $M$ of AdG-type satisfying the condition $\operatorname{tr}\left(\Omega^{2 m}\right)=\sigma$, then there exists a connection on $P$ with $\sigma$ as its Pontrjagin form.

As a direct consequence of the above theorem and Corollary 1.2.3 of [2], we get

Corollary 1.2. If a volume form $\sigma$ on $M$ can be realised as a Pontrjagin form of some connection on $P$, then every form in its cohomology class can also be realised by a connection.

The proof of the theorem employs sheaf theoretic techniques [1, Section 2.2] in $h$-principle theory. We extensively use the terminology and results of [1] throughout the article. For the sake of completeness we give a brief review of sheaf techniques in the Appendix.

Received by the editors February 10, 1999.

2000 Mathematics Subject Classification. Primary 58J99, 53C23, 53C05.

Key words and phrases. Principal bundles, connections, Pontrjagin class, $h$-principle.

This work was supported by the Commission on Development and Exchange of the International Mathematical Union. 
In this problem, the sheaf in question is the solution sheaf $\Phi$ of the equation $\operatorname{tr}\left((D \omega)^{2 m}\right)=\sigma$. To apply sheaf techniques it is desirable that this solution sheaf be microflexible (Definition 6.3). This is not the case here; but we find an auxiliary sheaf $\Phi_{0}$ closely associated with $\Phi$ which is microflexible. Indeed, we observe that $\Phi_{0}$ arises as the solution sheaf of an equation $\mathcal{D}=0$, for some infinitesimally invertible partial differential operator $\mathcal{D}$. Then, it follows from the Nash-Moser Implicit Function Theorem that $\Phi_{0}$ is microflexible. On the other hand, the class of ' $\sigma$-exact diffeotopies' on $M$ act on the sheaf $\Phi_{0}$. Moreover, these diffeotopies 'sharply moves a submanifold (of positive codimension) at hypersurfaces' (Definition 6.6). These facts about $\Phi_{0}$ set the problem in the required framework.

This article originates in an effort to answer a more general question raised by M. Gromov. He asks the same question that we deal with in this paper, but for all Pontrjagin classes. For some obvious reasons, which will be evident from the proof, we restrict ourselves only to the top-dimensional Pontrjagin class of a principal bundle over an open manifold. In other cases the admissible connection should also satisfy some regularity condition as explained in Section 3. We will treat the general problem in another paper.

\section{Preliminaries}

Let $G$ denote the orthogonal group $O(n)$ and let $P \longrightarrow M$ be a principal $G$ bundle. For any connection form $\omega$ on $P$ the $m$-th Pontrjagin form may be defined by

$$
\mathcal{P}(\omega)=\operatorname{tr}\left(\Omega^{2 m}\right),
$$

where $\Omega$ denotes the curvature form associated with the connection $\omega$. It follows from the general theory of connections that the cohomology class of the form $\mathcal{P}(\omega)$ does not depend on the choice of connection.

Indeed, if $\omega_{0}$ and $\omega_{1}$ are two connections on $P$, then they differ by a $\mathfrak{g}$-valued 1-form $\alpha$ on $P$ satisfying the following properties [2]:

1. $\alpha$ vanishes on the vertical vectors on $P$,

2. $\alpha$ is of type $A d G$. This means that $R_{a}^{*} \alpha=A d\left(a^{-1}\right) \alpha$ for all $a \in G$.

Hence $\alpha$ may be identified with a 1 -form on $M$ with values in the vector bundle $A d P$. We shall denote this space by $\mathcal{A}^{1}(A d P)$. Consequently, the space of connections is an affine space modelled on the vector space $\mathcal{A}^{1}(A d P)$, and the tangent space at any point is nothing but $\mathcal{A}^{1}(A d P)$. Also the curvature form $D \omega$ of a connection $\omega$ can be identified with a 2 -form on $M$ with values in $A d P$. Now $\mathcal{P}$ can be thought of as a differential operator on $\mathcal{A}^{1}(A d P)$ into the space $\mathcal{A}^{4 m-1}(M)$ of differential $(4 m-1)$-forms on $M$. Observe that here $\mathcal{P}$ is a first order differential operator on the space of sections of a vector bundle.

Let us now fix a connection $\omega^{0}$ on $P$. Then for any $\alpha$ in $\mathcal{A}^{1}(\operatorname{Ad} P)$ we have the following relation [2]:

$$
\mathcal{P}\left(\omega^{0}+\alpha\right)=\mathcal{P}\left(\omega^{0}\right)+d \int_{0}^{1} f\left(\alpha, \Omega_{t}\right) d t
$$

where $f\left(\alpha, \Omega_{t}\right)=\operatorname{tr}\left(\alpha \wedge \Omega_{t}^{2 m-1}\right)$ and $\Omega_{t}=D\left(\omega^{0}+t \alpha\right)$. Now it is easy to see that $f$ is $A d G$ invariant and hence $f\left(\alpha, \Omega_{t}\right)$ projects onto a $(4 m-1)$-form on $M$. By an abuse of notation, we also denote the projected form by the same symbol. 
We now define an auxiliary differential operator as follows:

$$
\mathcal{D}_{\omega^{0}}(\alpha, \varphi)=\int_{0}^{1} f\left(\alpha, \Omega_{t}\right) d t+d \varphi
$$

Here, the integral part comes from the above expression for $\mathcal{P}\left(\omega^{0}+\alpha\right)$ and $\varphi$ is a differential $(4 m-2)$-form on $M$. With the introduction of this notation, equation (1) reads as

$$
\mathcal{P}\left(\omega^{0}+\alpha\right)=\mathcal{P}\left(\omega^{0}\right)+d \mathcal{D}_{\omega^{0}}(\alpha, \varphi) .
$$

We shall drop $\omega^{0}$ in the notation of the operator $\mathcal{D}_{\omega^{0}}$ and simply denote it by $\mathcal{D}$. Also, we denote $\mathcal{D}(\alpha, 0)$ by $\mathcal{D}(\alpha)$.

Let $\mathcal{P}\left(\omega^{0}\right)=\sigma_{0}$ and let $\sigma=\sigma_{0}+d \tau$. Then it follows from relation (3) that there is a canonical morphism from the sheaf $\mathcal{D}=\tau$ into $\mathcal{P}=\sigma_{0}+d \tau$. On the other hand, if $\mathcal{P}\left(\omega^{0}+\alpha\right)=\sigma_{0}+d \tau$, then

$$
\mathcal{D}(\alpha)=\int_{0}^{1} f\left(\alpha, D \omega_{t}\right) d t=\tau+\text { a closed form. }
$$

We denote the sheaf of solutions of $\mathcal{P}=\sigma_{0}+d \tau=\sigma$ by $\Phi$ and that of solutions of $\mathcal{D}=\tau$ by $\Phi_{0}$. Then the canonical map $\pi(x): \Phi(x) \longrightarrow \Phi_{0}(x)$ is a surjection, because on an appropriate neighbourhood of $x$ a closed form is always exact. The sheaves $\Phi$ and $\Phi_{0}$ come with the quasi-topological structure (Definition 6.2) associated with the $C^{\infty}$ compact open topologies on the concerned spaces.

As Gromov has observed [1 3.4.1(B)], the sheaf $\Phi$ cannot be microflexible. However, we shall conclude that the sheaf $\Phi_{0}$ is microflexible by observing that the differential operator $\mathcal{D}$ is infinitesimally invertible at 'regular' connections, and hence the Nash-Moser Implicit Function theorem applies to the situation.

\section{INFINITESIMAL INVERSION OF $\mathcal{D}$ AND MICROFLEXIBILITY OF $\Phi_{0}$}

We begin with the following

Definition 3.1. A connection $\omega$ on a principal bundle $P$ is said to be regular at a point $x$ if the linear map

$$
\alpha_{x} \mapsto f\left(\alpha_{x}, D \omega_{x}\right)
$$

is surjective, where $\alpha$ is an $A d P$-valued 1-form on $M$ and $\alpha_{x}$ is its value at the point $x$. A connection will be called regular if it is regular at every point.

It is clear from the definition that regularity conditions define a 1-st order open differential relation on the space of connections.

Note that flat connections are not regular.

Proposition 3.2. The operator $\mathcal{D}$ is infinitesimally invertible at regular connections.

Proof. It follows from the definition of $\mathcal{D}$ (see Equation 2) that

$$
\mathcal{D}(\alpha+s \beta)=\int_{0}^{1} f(\alpha+s \beta, D(\omega+t \alpha+t s \beta)) d t
$$

where $\alpha, \beta$ are sections of $\mathcal{A}^{1}(A d P)$. Differentiating the above relation with respect to $s$ at the point $s=0$, we get

$$
\left.\frac{d}{d s} \mathcal{D}(\alpha+s \beta)\right|_{s=0}=f(\beta, D(\omega+\alpha))+d \psi_{\omega, \alpha, \beta},
$$


where $\psi_{\omega, \alpha, \beta}$ denotes a $(4 m-2)$-form on $M$ which depends on $\omega, \alpha$ and $\beta$ [4] Equation 4.2.41]. Therefore, the linearization of the differential operator is given by:

$$
d \mathcal{D}_{\alpha, \varphi}(\beta, \psi)=f(\beta, D(\omega+\alpha))+d \psi_{\omega, \alpha, \beta}+d \psi .
$$

Now, for any $(4 m-1)$-form $\tau, d \mathcal{D}_{\omega+\alpha, \varphi}(\beta, \psi)=\tau$ can be solved for $(\beta, \psi)$ as follows:

$$
\begin{aligned}
& f(\beta, D(\omega+\alpha))=\tau, \\
& \psi_{\omega, \alpha, \beta}+\psi=0,
\end{aligned}
$$

provided $\omega+\alpha$ is a regular connection. This proves the infinitesimal invertibility of the operator $\mathcal{D}$ at regular connections.

Observation. Since the differential form $\sigma$ in Theorem 1.1 is a volume form, $M$ is $\sigma$-regular 1, 3.4.1(A)]; that is, any $(4 m-1)$-form $\tau$ on $M$ comes from a vector field on $M$ in the following way: $X . \sigma=\tau(X . \sigma$ denotes the interior product of the form $\sigma$ with the vector field $X$ ). Hence, if $\omega$ is a connection on $P$ such that $\mathcal{P}(\omega)=\sigma$, then the above relation tells us that the horizontal lift $\widetilde{X}$ of $X$ satisfies the relation $f(\widetilde{X} . \Omega, \Omega)=\tau$ which clearly implies that $\omega$ is a regular connection. Thus, any connection $\omega$ which satisfies $\mathcal{P}(\omega)=\sigma$ is automatically a regular connection.

It then follows from the Nash-Moser Implicit Function Theorem ([1, 2.3.2]) that the operator $\mathcal{D}$ is locally invertible and hence

Theorem $3.3\left(\left[1,2.3 .2\left(\mathrm{D}^{\prime \prime}\right)\right]\right) . \Phi_{0}$ is a microflexible sheaf.

\section{EXACT DIFFEOTOPY ACTION ON $\Phi_{0}$}

The volume form $\sigma$ on $M$ defines a bundle isomorphism $\widetilde{\sigma}: T M \longrightarrow \Lambda^{4 m-1} T^{*} M$ by the formula $X \mapsto X . \sigma$. If $X$ is vector field on $M$ which corresponds to an exact form under the above isomorphism, then following the language of Gromov we call such a vector field exact [1, 3.4.1(B)]. Integrating the vector field $X$ we obtain a 1-parameter family of diffeomorphisms which preserve the volume form $\sigma$. Such a diffeotopy will be called a $\sigma$-exact diffeotopy. It may be seen that

Proposition 4.1. $\sigma$-exact diffeotopies sharply move a submanifold $N$ at hypersurfaces.

Proof (Compare [1,3.4.2]). Let $S$ be a hypersurface in $N$ and let $m \in S$. Any tangent vector $X_{m} \in T_{m} M$ which is transversal to the given submanifold $N$ can be extended locally to a $\sigma$-exact vector field. We multiply this vector field by a suitably chosen function defined on a neighbourhood of $x$, which vanishes outside a given neighbourhood of $S$ and takes a constant positive value on a neighbourhood of $S$. We then integrate the resulting vector field to obtain an isotopy which satisfies the required property.

Let $\delta_{s}, 0 \leq s \leq 1$, be a $\sigma$-exact isotopy on $M$; that is, $\delta_{0}=i d$ and $X_{s}=\frac{d}{d s} \delta_{s}$ satisfy the relation $X_{s} . \sigma=d \alpha_{s}$ for a 1-parameter family of $(4 m-2)$-forms $\alpha_{s}$ on $M$. We denote the horizontal lift of $X_{s}$ by $\tilde{X}_{s}$.

Let $\omega$ be a connection on $P$ such that $\mathcal{P}(\omega)=\sigma$. Let $\omega_{s}=\bar{\delta}_{s}^{*} \omega$, where $\left\{\bar{\delta}_{s}\right\}$ is the 1-parameter family of diffeomorphisms on $P$ obtained by integrating $\left\{\widetilde{X}_{s}\right\}$. Then $\mathcal{P}\left(\omega_{s}\right)=\delta_{s}^{*} \mathcal{P}(\omega)=\sigma$. Moreover, we get that $f\left(\widetilde{X}_{s} . \Omega_{s}, \Omega_{s}\right)=X_{s} \cdot \mathcal{P}\left(\omega_{s}\right)=X_{s} . \sigma=$ $d \alpha_{s}$ is an exact form on $M$ for every $s$. We now prove 
Proposition 4.2. The sheaf of solutions of $\mathcal{D}=\tau$ is acted upon by $\sigma$-exact diffeotopies of $M$.

Proof. Differentiating $\bar{\delta}_{s}^{*} \omega$ with respect to $s$, we get

$$
\begin{aligned}
\frac{d}{d s} \bar{\delta}_{s}^{*} \omega & =\widetilde{X}_{s} \cdot d \omega_{s}+d\left(\widetilde{X}_{s} \cdot \omega_{s}\right) \\
& =\widetilde{X}_{s} \cdot D \omega_{s}-\frac{1}{2} \widetilde{X}_{s} \cdot\left[\omega_{s}, \omega_{s}\right]+d\left(\widetilde{X}_{s} \cdot \omega_{s}\right) \\
& =\widetilde{X}_{s} \cdot \Omega_{s}-\left[\widetilde{X}_{s} \cdot \omega_{s}, \omega_{s}\right]+d\left(\widetilde{X}_{s} \cdot \omega_{s}\right) \\
& =\widetilde{X}_{s} \cdot \Omega_{s}+D\left(\widetilde{X}_{s} \cdot \omega_{s}\right) .
\end{aligned}
$$

Writing $\omega_{s}=\omega^{0}+\alpha_{s}$ and using the relation (4.2.41) in [3] we get

$$
\begin{aligned}
\frac{d}{d s} \mathcal{D}_{\omega^{0}}\left(\alpha_{s}\right) & =f\left(\frac{d}{d s} \omega_{s}, \Omega_{s}\right)+\text { exact forms on } M \\
& =f\left(\widetilde{X}_{s} \cdot \Omega_{s}, \Omega_{s}\right)+f\left(D\left(\widetilde{X}_{s} \cdot \omega_{s}\right), \Omega_{s}\right)+\text { exact forms on } M \\
& =f\left(\widetilde{X}_{s} \cdot \Omega_{s}, \Omega_{s}\right)+d f\left(\widetilde{X}_{s} \cdot \omega_{s}, \Omega_{s}\right)+\text { exact forms on } M \\
& =\text { an exact forms on } M
\end{aligned}
$$

since $f\left(\widetilde{X}_{s} . \Omega_{s}, \Omega_{s}\right)$ is exact. Hence $\mathcal{D}\left(\alpha_{s}\right)=\mathcal{D}\left(\alpha_{0}\right)+$ exact forms. This completes the proof.

We now conclude from Theorem 3.3 and Propositions 4.1 and 4.2 that the sheaf $\left.\Phi_{0}\right|_{N}$ is flexible (see Theorem 6.7).

\section{Proof of the MAIN THEOREM}

Let $\mathcal{R}$ denote the differential relation consisting of 1 -jets of germs of connections $\omega$ at $x$ such that $\mathcal{P}(\omega)(x)=\sigma, x \in X$. Then

Lemma 5.1. $\mathcal{R}$ satisfies local h-principle.

Proof. It is enough to show that an infinitesimal solution of $\mathcal{R}$ can be homotoped to a local solution of $\mathcal{R}$; that is, if $\omega$ is a connection which satisfies the relation $\mathcal{P}(\omega)=\sigma$ at $x$, then we can homotope it to a local solution of the differential equation $\mathcal{P}=\sigma$ near $x$ by keeping it constant at $x$. Indeed, for such an $\omega$ we have $\mathcal{P}(\omega)-\sigma=d \varphi$ for some $(4 m-1)$-form $\varphi$ near $x$ such that $\varphi(x)=0$ and $d \varphi_{x}=0$. We now apply the Moser-type argument. Consider the family of $4 m$-forms $\left\{\sigma_{t}\right\}$ defined by $\sigma_{t}=\sigma+t d \varphi, t \in[0,1]$. Since $\sigma_{t}(x)=\sigma(x)$ for all $t$ (and since $\sigma$ regularity condition is open) we observe that $M$ is $\sigma_{t}$-regular on a neighbourhood of $x$ for each $t$. Now, by the observation of Section 3, we get a family of vector fields $X_{t}$ on $M$ such that $X_{t} \cdot \sigma_{t}=-\varphi$ and $X_{t}(x)=0$. We integrate $\left\{X_{t}\right\}$ to obtain a 1-parameter family of (local) diffeomorphisms $\left\{\delta_{t}\right\}$ such that $\frac{d}{d t} \delta_{t}=X_{t}$ and $\delta_{t}(x)=x, d \delta_{t}(x)=i d$. It is now easy to see that $\delta_{t}^{*} \sigma_{t}=\sigma$ for all $t$. In particular, $\delta_{1}^{*} \mathcal{P}(\omega)=\sigma$. This isotopy can be lifted locally to an equivariant isotopy $\bar{\delta}_{t}$ on $P$. Since the pair $\left(\bar{\delta}_{t}, \delta_{t}\right)$ intertwine with the projection map $p: P \longrightarrow M$, we have $\mathcal{P}\left(\bar{\delta}_{1}^{*} \omega\right)=\delta_{1}^{*} \mathcal{P}(\omega)=\sigma$. Thus $\bar{\delta}_{1}^{*} \omega$ is a local solution of $\mathcal{P}=\sigma$ and also $D\left(\bar{\delta}_{1}^{*} \omega\right)=D \omega$ at $x$. This completes the proof.

Since we have already proved that $\Phi_{0} \mid N$ is flexible, we now conclude that $\Phi_{0} \mid N$ satisfies the $h$-principle (Theorem 6.5).

Observation. The space of sections of $\mathcal{R}$ has the same homotopy type as the space of $\mathfrak{g}$-valued (continuous) antisymmetric 2 -forms $\Omega$ on $M$ of $A d G$-type such that $\operatorname{tr}(\Omega)^{2 m}=\sigma$. 
Thus, if we have an $\Omega$ as in Theorem 1.1, then there exists a neighbourhood $U$ of $N$ and a connection $\omega$ on $\left.P\right|_{U}$, which has the Pontrjagin form $\left.\sigma\right|_{U}$. Here $N$ is a submanifold of $M$ of positive codimension. In fact, we can, and we do, take $N$ to be the $(4 m-1)$-skeleton of $M$. We now construct a connection defined over all of $P$ which has the desired property. Since $M$ is open, it can be isotoped into an arbitrary neighbourhood of its $(4 m-1)$-skeleton, in particular into the neighbourhood $U$ of the skeleton $N$ over which $\omega$ has already been constructed. Let $f_{0}: M \longrightarrow U \subset M$ be the final map of the isotopy. By a result of Gromov and Eliashberg [2, 1.2.2], we obtain a $\sigma$-preserving map $f: M \longrightarrow U$ which is homotopic to $f_{0}$. Consider the pull-back connection $f^{*} \omega$ on $f^{*} P$. The Pontrjagin form corresponding to this connection is easily seen to be $\sigma$. Since $f$ is homotopic to the identity map, $f^{*} P$ is isomorphic to $P$ as principal $G$ bundle and our result follows for the case when $\sigma$ is a volume form.

Example. Let $(M, \sigma)$ be an open symplectic manifold of dimension $n=4 \mathrm{~m}$ and let $P$ be a trivial principal $O(2 m)$ bundle on $M$. Then every exact $n$-form on $M$ is realisable as a Pontrjagin form on $P$. Indeed, the form $\sigma^{2 m}$ is an exact volume form and we have $p(P)=\left[\sigma^{2 m}\right]$. Consider the $\mathfrak{o}(2 m)$-valued antisymmetric 2 -form $\Omega$ defined as follows:

$$
\Omega_{i j}= \begin{cases}\sigma & \text { if } i>j \\ -\sigma & \text { if } i<j \\ 0 & \text { if } i=j\end{cases}
$$

It is easy to see that $\operatorname{tr}\left(\Omega^{2 m}\right)=\sigma^{2 m}$. Thus it follows from Theorem 1.1 and Corollary 1.2 that every exact form on $M$ is a Pontrjagin form of some connection on $P$.

\section{Appendix}

Here we briefly describe the sheaf theoretic techniques in $h$-principle.

Let $p: E \longrightarrow M$ be a $C^{\infty}$ fibration and let $p^{(r)}: E^{(r)} \longrightarrow M$ denote the associated $r$-jet bundle for sections of $E$. A subset $\mathcal{R} \subset E^{(r)}$ is called an $r$-th order partial differential relation for sections of $p$. A section $f$ of $p$ is said to be a solution of $\mathcal{R}$ if its $r$-jet map $\jmath^{r} f$ maps $M$ into $\mathcal{R}$. We denote the solution space by Sol $\mathcal{R}$ and endow it with $C^{\infty}$-compact open toplogy. On the other hand, we denote by $\Gamma \mathcal{R}$ the space of (continuous) sections of $p^{(r)}$ whose images lie in $\mathcal{R}$. This space comes with the $C^{0}$ compact open toplogy. A section of $p^{(r)}$ is called holonomic if it is the $r$-jet map of a solution of $\mathcal{R}$.

Definition 6.1. A relation $\mathcal{R}$ is said to satisfy the $h$-principle if a section of $\mathcal{R}$ can be homotoped in $\mathcal{R}$ to a holonomic section.

$\mathcal{R}$ satisfies the parametric $h$-principle if the $r$-jet map $\jmath^{r}: \operatorname{Sol} \mathcal{R} \longrightarrow \Gamma \mathcal{R}$ is a weak homotopy equivalence.

Let $\Phi$ be the sheaf of solutions of $\mathcal{R}$, and $\Psi$ the sheaf of sections of $\mathcal{R}$. The topologies on $\Phi(U)$ and $\Psi(U)$ are respectively the $C^{\infty}$ and $C^{0}$ compact open topologies.

Before we state a theorem concerning the $h$-principle of $\mathcal{R}$, we recall some general definitions and terminology from [1].

Definition 6.2. Let $\mathcal{F}$ be a topological sheaf over $M$ and $A$ a compact set in $M$. Then the symbol $\mathcal{F}(A)$ will denote the space of maps which are defined over some 
neighbourhood of $A$ in $M$; in fact it is the direct limit of the spaces $\mathcal{F}(U)$ where $U$ runs over all the open sets containing $A$. A map $f: P \longrightarrow \mathcal{F}(A)$ on a polyhedron $P$ is called continuous if there exists an open set $U \supset A$ such that each $f_{p}$ is defined over $U$ and the resulting map $P \longrightarrow \mathcal{F}(U)$ is continuous with respect to the given topology on $\mathcal{F}(U)$.

Definition 6.3. A topological sheaf $\mathcal{F}$ over $M$ is flexible if the restriction maps $\mathcal{F}(A) \longrightarrow \mathcal{F}(B)$ are Serre fibrations for every pair of compact sets $(A, B), A \supset B$. The restriction map $\mathcal{F}(A) \longrightarrow \mathcal{F}(B)$ is called a microfibration if given a continuous map $f_{0}^{\prime}: P \longrightarrow \mathcal{F}(A)$ on a polyhedron $P$ and a homotopy $f_{t}, 0 \leq t \leq 1$, of $\left.f_{0}^{\prime}\right|_{B}$ there exists an $\varepsilon>0$ and a homotopy $f_{t}^{\prime}$ of $f_{0}^{\prime}$ such that $f_{t}^{\prime} \mid \mathrm{Op} B=f_{t}$ for $0 \leq t \leq \varepsilon$. If for any pair of compact sets the restriction morphism is a microfibration, then the sheaf $\mathcal{F}$ is called microflexible.

Definition 6.4. $\mathcal{R}$ satisfies the local parametric h-principle if for each $x \in M$, $f^{r}(x): \Phi(x) \longrightarrow \Psi(x)$ is a weak homotopy equivalence.

We now state the main theorem on the $h$-principle for a relation $\mathcal{R}$.

Theorem 6.5 ([1, p. 77]). If the solution sheaf $\Phi$ is flexible and if $\mathcal{R}$ satisfies the local parametric $h$-principle, then $\mathcal{R}$ satisfies the parametric $h$-principle.

The most crucial part in proving the $h$-principle is to establish flexibility of the solution sheaf $\Phi$. In many cases it can be easily seen that the solution sheaf is microflexible. Moreover, if we have a class of 'sharp diffeotopies' which act on the solution sheaf $\Phi$, then Gromov proves in his Main Lemma ([1, 2.2.3(C')]) that for any embedded submaniflod $N$ of positive codimension, $\Phi \mid N$ is flexible. Before we state his result we explain the concept of diffeotopy sharply moving a submanifold $N$ in $M$.

Definition 6.6. We fix a metric $d$ on $M$. An open set in $M$ will be called 'small' if it is contained in a ball of small radius. A class of diffeotopies $\mathcal{D}$ on $M$ is said to sharply move $N$ in $M$ if given any hypersurface $S$ lying in a small open set of $M$ and given any positive numbers $\varepsilon$ we can obtain a diffeotopy $\left\{\delta_{t}\right\}$ in $\mathcal{D}$ which satisfies the following conditions:

(a) $\delta_{0}$ is the identity map,

(b) each $\delta_{t}$ is the identity outside an $\varepsilon$-neighbourhood of $S$ in $M$,

(c) $d\left(\delta_{1}(S), M\right)>r$ for some $r>0$.

Theorem 6.7. If the solution sheaf $\Phi$ is microflexible and if there exists a class of acting diffeotopy on $\Phi$ which sharply moves $N$ in $M$, then $\Phi \mid N$ is a flexible sheaf.

\section{REFERENCES}

[1] Mikhael Gromov: Partial Differential relations, Ergebnisse der Mathematik und ihrer Grenzgebiete 3 Folge, Band 9 (1986), Springer Verlag. MR 90a:58201

[2] M. Gromov and Ya. Eliashberg: Construction of a Smooth Mapping with a Prescribed Jacobian. I, Func. Anal. and Applications 7(1973), pp.423-434. MR 50:5841

[3] S. Kobayashi, K. Nomizu: Foundations of Differential Geometry (v. 1, 2) Interscience Tracts in Pure and Applied Mathematics (1969), John Wiley and Sons. MR 38:6501 MR 97c:53001a

[4] Izu Vaisman: Symplectic Geometry and Secondary Characteristic Classes, Progress in Mathematics 72 (1987), Birkhäuser. MR 89f:58062

Department of Pure Mathematics, University College of Science, 35, P.Barua Sarani, Calcutta 700019, India

E-mail address: mahuyad@hotmail.com 